\title{
Effect of needle diameter, type and volume of contrast agent on intervertebral disc degeneration in rats with discography
}

\author{
Xiaodong Huang ${ }^{1} \cdot$ Weiheng Wang $^{1} \cdot$ Qingxi Meng $^{1} \cdot$ Lei $\mathrm{Yu}^{1} \cdot$ Chunquan Fan ${ }^{2} \cdot$ Jiangming $\mathrm{Yu}^{1} \cdot$ Mintao Xue $^{1}$. \\ Xiaojian $\mathrm{Ye}^{1}$ (1)
}

Received: 29 May 2018 / Revised: 17 January 2019 / Accepted: 21 February 2019 / Published online: 12 March 2019

(c) The Author(s) 2019

\begin{abstract}
Purpose Discography can increase disc degeneration, but the influence of different discography variables on the degeneration of discs has not been reported. The aim of this study was to investigate the effects of discography variables of needle diameter, type of contrast agent and volume of contrast agent on disc degeneration.

Methods Three separate experiments examined needle diameter, and type and volume of contrast agent. Coccygeal discs (Co7-10) adult male rats were used. X-rays were used to detect the disc height degeneration index at 1, 2 and 4 weeks after the procedure. MRI was used to study the changes in the disc structure and the signal intensity of IVD 2 and 4 weeks after the procedure. Disc water content and histology were measured at 4 weeks after the procedure.

Results A 21-g needle significantly increased disc degeneration when compared with the 30-g needle as detected by X-ray, MRI, disc water content and histology $(P<0.05)$. Two microlitres of iodine significantly decreased the disc MRI signal and water content at 4 weeks compared with the same volume of normal saline $(P<0.05)$. Three microlitres of iodine significantly increased disc degeneration when compared with $2 \mu \mathrm{l}$ iodine, as detected by X-ray, MRI, disc water content and histology at 4 weeks $(P<0.05)$. Conclusion To reduce disc degeneration after discography, it may be best to choose a smaller needle size, minimize the use of contrast agent and use non-ionic contrast agents with osmotic pressure similar to the intervertebral disc.
\end{abstract}

\section{Graphical abstract}

These slides can be retrieved under Electronic Supplementary Material.
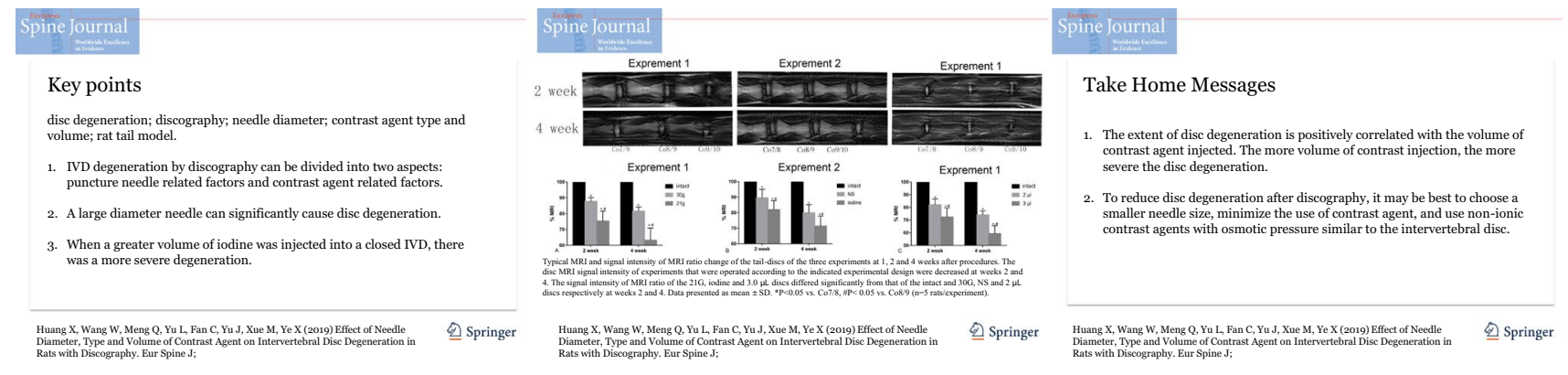

Keywords Disc degeneration · Discography $\cdot$ Needle diameter $\cdot$ Contrast agent type and volume $\cdot$ Rat tail model

Mintao Xue and Xiaojian Ye supported this project equally.

Xiaodong Huang, Weiheng Wang and Qingxi Meng contributed equally to this work.

Electronic supplementary material The online version of this article (https://doi.org/10.1007/s00586-019-05927-0) contains supplementary material, which is available to authorized users.

Extended author information available on the last page of the article

\section{Introduction}

Discography was first introduced by Lindbloom in 1948, and the accurate appearance of disc shape and evoked pain are important for definite diagnosis. Surgical localization makes discography an invasive diagnostic technique with irreplaceable advantages: typical pain reproduction, fine 
and intuitive display of intervertebral disc (IVD) injury for diagnosing low back pain [1]. At present, there is no accurate quantitative standard for the injection metering of discography, and there has been a great deal of controversy since the beginning of the invention [2]. More and more attention has been paid to the occurrence and acceleration of IVD degeneration caused by discography. Over 10 years of clinical studies have shown that discography causes accelerated progression of degeneration changes in the lumbar disc $[3,4]$. A recent in vivo study showed that discography and disc block could lead to cell toxicity and apoptosis [5]. In vitro cell experiments have shown that local anaesthetics and non-ionic contrast are cytotoxic to annulus fibrosis cells and nucleus pulposus cells [6].

The factors affecting IVD degeneration by discography can be divided into two aspects: puncture needle-related factors and contrast agent-related factors. Studies have shown that disc puncture can cause IVD degeneration [7-11]. The IVD degeneration caused by disc puncture has been widely used in animal models, because it is simple, rapid and reproducible [12-14]. However, there is controversy about the IVD degeneration caused by the diameter of the puncture needle. Some people think that small-diameter needles do not cause degeneration of the intervertebral disc, and some studies suggest that even small needles will accelerate the degeneration of the intervertebral disc. A system review has shown that the needle height between $25 \%$ and $40 \%$ results was variable and no significant effects were observed [7]. Studies have also shown that significant intervertebral image changes by MRI were observed when the IVD degeneration models are produced using 30-g needles (less than 25\% intervertebral height) [15]. So, a single systematic in vivo study is needed to address the effect of the diameter of the needle on the degeneration of the intervertebral disc. Delamination injury as induced from puncture and injection of saline was also shown to result in degeneration and biomechanical changes, and warrants additional investigation since injection with saline (in addition to contrast agent) can cause greater damage than puncture alone [16]. Contrast agents are another important factor that affects the IVD degeneration. The effect of contrast on IVD degeneration is divided into two aspects: the type and dose of contrast agent. Iodine is the second generation of non-ionic imaging agents and widely used in clinics [17]. However, cell experiments have shown that iodine is cytotoxic to annulus fibrosis cells, nucleus pulposus and end plate chondrocytes $[5,6,18]$. The effect of contrast agent type and dose on the degeneration of IVD in vivo has not been reported.

The aim of this study was to investigate the effect of puncture needle diameter and contrast agent type and volume on IVD degeneration after discography in rats and provide a theoretical and experimental basis for assessing the value and risk of clinical discography.

\section{Methods and materials}

\section{Experimental animals}

Three-month-old Sprague-Dawley (SD) rats (male, skeletal maturity at this age) [19] were purchased from the experimental Animal Center of Shanghai Second Military Medical University. Rats were housed at temperature of $25 \pm 1{ }^{\circ} \mathrm{C}$ with a 12-h light-dark cycle and free access to food and water. All animal operations were approved by the animal ethics committee of the Second Military Medical University.

\section{Experimental design and the establishment of rat models}

Rats were anesthetized by $1.5 \%$ isoflurane. Coccygeal discs (Co7-10) of tails were used, with Co 7/8 disc remaining intact. The experiment design is shown in Fig. 1. A total of 30 rats were randomly divided into three equal experiments. Experiment 1 ( $n=10$ rats) was used to assess the effect of needle diameter. A 30 gauge $(\mathrm{g})$ needle was used to inject $2 \mu \mathrm{l} \mathrm{NS}$ into Co 8/9. A 21 -g needle was used to inject $2 \mu \mathrm{NS}$ into Co $9 / 10$. Experiment 2 ( $n=10$ rats) was used to assess the effect of the type of contrast agent. A 30-g needle was used to inject $2 \mu \mathrm{lNS}$ into Co 8/9. A 30-g needle was used to inject $2 \mu$ iodine (General Electric Pharmaceutical, Shanghai, $300 \mathrm{mg} \mathrm{I} / \mathrm{ml}, 780 \mathrm{mOsm} / \mathrm{kg}$ ) into Co 9/10. Experiment 3 ( $n=10$ rats) was used to assess the effect of the volume of contrast agent. A 30-g needle was used to inject $2 \mu \mathrm{l}$ iodine into Co 8/9, and a 30-g needle was used to inject $3 \mu$ iodine into Co $9 / 10$. These procedures were carried out under X-ray guidance, and the location of the needle was in the centre of the disc according to the technique described previously (Supplementary Fig. 1) [20]. NS or iodine was injected by using an insulin micro-injector (Hamilton, Switzerland) over at least 5 min to prevent liquid leakage.

\section{X-ray}

One, 2 and 4 weeks after the procedure, the rats were anesthetized to keep the caudal muscle relaxed. The rat tail disc was scanned by X-ray. The disc height index (DHI) measurement was carried out according to Lin (Supplementary Fig. 1) [21]. All images were measured by two independent observers who were blinded to the specimens. The changes in DHI were analysed by Sante DICOM Viewer FREE image software $(n=10)$. 
Fig. 1 Experiment design

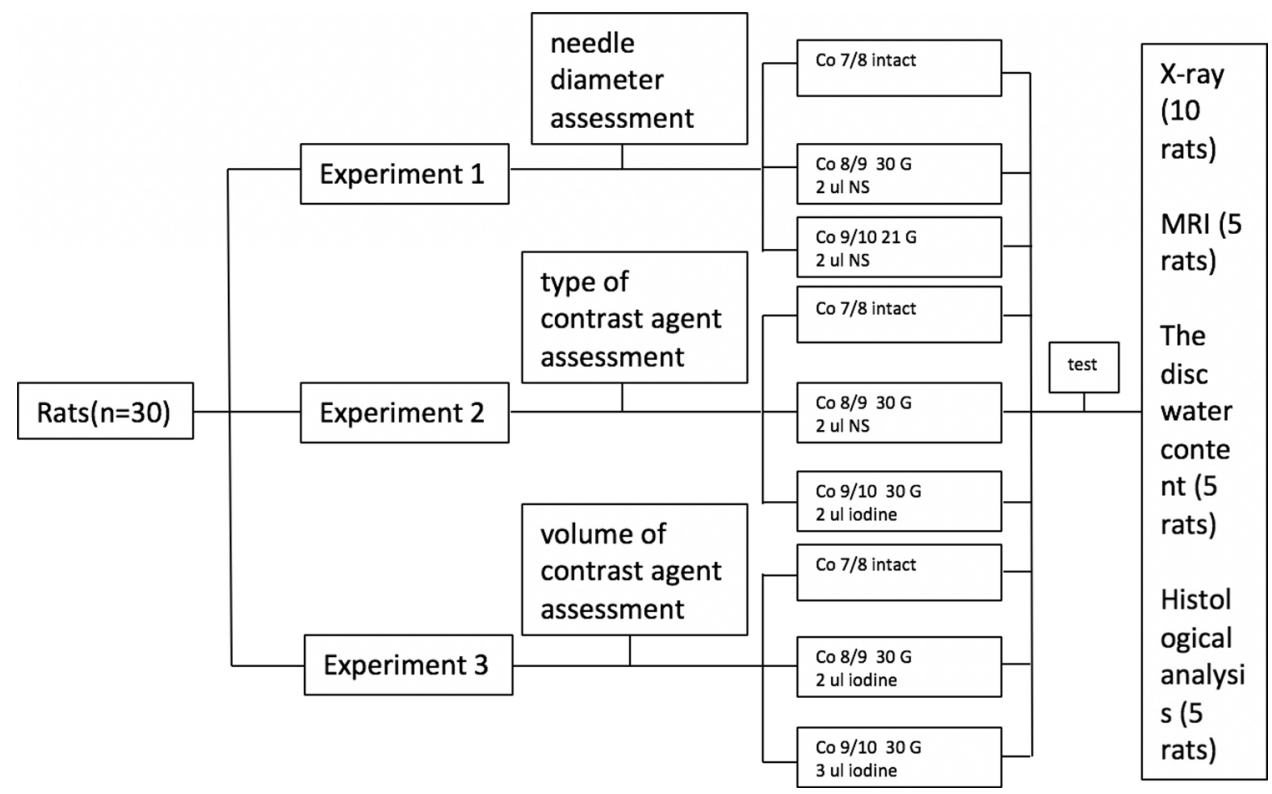

MRI

MRI was used to study the changes in the disc structure and the signal intensity of IVD 2 and 4 weeks after the procedure. In half of the rats ( $n=5 /$ experiment), the rat tail discs were scanned using a small animal MRI scanner of $3.0 \mathrm{~T}$ (Philips ${ }^{\circledR}$, Achieva, The Netherlands) as described in a previous article [20]. The signal strength of the T2-weighted image was used to assess the IVD degeneration. IVD were analysed using ImageJ software. In order to evaluate the entire disc, five sequential sagittal images of IVD were obtained, and the mean pixel intensity value was quantified [22]. This was measured by two independent observers who were blinded to the specimens' treatment ( $n=5$ rats).

\section{The disc water content}

Four weeks after the procedure, all rats were euthanized by $\mathrm{CO}_{2}$. In half of the rats ( $n=5 /$ experiment), Co7-10 discs were separated from the surrounding soft tissue using a scalpel. Disc wet weight was recorded using an electronic scale with an accuracy of $0.01 \mathrm{mg}$. Then, the samples were dehydrated at $65{ }^{\circ} \mathrm{C}$ for $48 \mathrm{~h}$, and disc dry weight was recorded using the same scale. Wet and dry weights were measured three times, and the mean disc water percentage was calculated ( $n=5$ rats).

\section{Histological analysis}

Four weeks after procedure, Co7-10 discs were harvested for histological analysis in the remaining five rats per experiment. These discs were fixed in formalin for at least $48 \mathrm{~h}$, and decalcified in the ETDA solution for $48 \mathrm{~h}$. After decalcification, the intervertebral disc was sliced by transverse, that is, parallel to the bilateral laminar cartilage. These sections $(5 \mu \mathrm{m})$ were stained with haematoxylin and eosin (HE, $n=5)$. The histological scale of HE was used to determine the cellular and morphological changes in both annulus fibrosus (AF) and nucleus pulposus (NP) cells according to the previously published paper (Supplementary Table 1) [13].

\section{Statistical Analysis}

Data analysis was performed with SPSS 21 software (SPSS Inc., Chicago, USA). All data are presented as mean \pm SD. Data were analysed by a one-way ANOVA followed by Student-Newman-Keuls analyses. $P<0.05$ was considered statistically significant.

\section{Results}

\section{The effect of needle diameter on disc degeneration in discography}

The 21-g needle significantly increased disc degeneration when compared with the $30 \mathrm{~g}$. Radiography imaging showed that there was no significant difference of DHI between the Co7/8 (intact) and Co8/9 (30 g). DHI ratio decreased significantly in the Co9/10 $(21 \mathrm{~g})$ compared with the Co7/8 (intact) and the Co8/9 (30 g) at 2 and 4 weeks after the procedure (Fig. 2 experiment 1 , $n=10$ rats, $P<0.05)$. The signal intensity of MRI ratio decreased significantly in both the Co8/9 $(30 \mathrm{~g})$ and the Co9/10 (21 g) compared with the Co7/8 (intact) discs at 2 and 4 weeks after the procedure. The ratio in the Co8/9 $(30 \mathrm{~g})$ was much higher than that in the Co9/10 (21 g) at 2 
and 4 weeks (Fig. 3 experiment $1, n=5$ rats, $P<0.05$ ). The signal intensities of MRI ratio in both the Co8/9 (30 g) and the Co9/10 (21 g) at 2 weeks were much higher than that at 4 weeks (Fig. 3, experiment $1, n=5$ rats, $P<0.05$ ). No significant differences of water content were observed between the Co7/8 (intact) and the Co8/9 (30 g) discs at 4 weeks. The Co9/10 (21 g) had a significantly lower disc water content than the Co8/9 (30 g) at 4 weeks (Fig. 4, experiment $1, n=5$ rats, $P>0.05$ ). HE staining showed morphological changes in the NP and the AF regions. According to histological scoring, there were no significant changes between the Co7/8 (intact) and the Co8/9 $(30 \mathrm{~g})$ discs at 4 weeks. The scoring of the Co9/10 (21 g) discs was graded as $3-5$, which was much higher than that in the Co7/8 (intact) and the Co8/9 (30 g) which were graded as $0-2$ (Fig. 5, experiment $1, n=5$ rats, $P<0.05$ ). These results demonstrate that IVD degenerative changes were needle diameter-dependent and increased as the needle diameter increased in discography.

\section{The effect of contrast agent type on disc degeneration in discography}

According to the X-ray and histology measurements, there was no significant difference in DHI (Fig. 2, experiment 2, $n=10$ rats) and histological scoring (Fig. 5 experiment 2, $n=5$ rats) among the Co7/8 (intact), the Co8/9 (NS) and the Co9/10 (iodine) discs $(P>0.05)$. The signal intensity of MRI ratio decreased significantly in both the Co8/9 (NS) and the Co9/10 (iodine) discs compared with the Co7/8 (intact) disc at 2 and 4 weeks after the procedure. The Co9/10 (iodine) signal intensity of MRI ratio was much lower than the Co8/9 (NS) at 2 and 4 weeks (Fig. 3 experiment $2, n=5$ rats, $P<0.05$ ). The signal intensity of MRI ratio showed that the effect of time after injury at each individual contrast agent type was statistically significant (Fig. 3, experiment $2, n=5$ rats, $P<0.05$ ). No significant differences of water content between the Co7/8 (intact) and the Co8/9 (NS) discs were observed at 4 weeks. The Co9/10 (iodine) disc had a significantly lower disc water content than the Co7/8 (intact) and the Co8/9 (NS) discs at 4 weeks (Fig. 4 experiment $2, n=5$ rats, $P<0.05$ ).

\section{The effect of contrast agent volume on disc degeneration in discography}

A higher contrast agent volume can significantly increase the disc degeneration as detected by X-ray, MRI, disc water content and histology also shown in experiment 3. Radiography imaging showed that there was no significant difference in DHI between the Co7/8 (intact) and the Co8/9

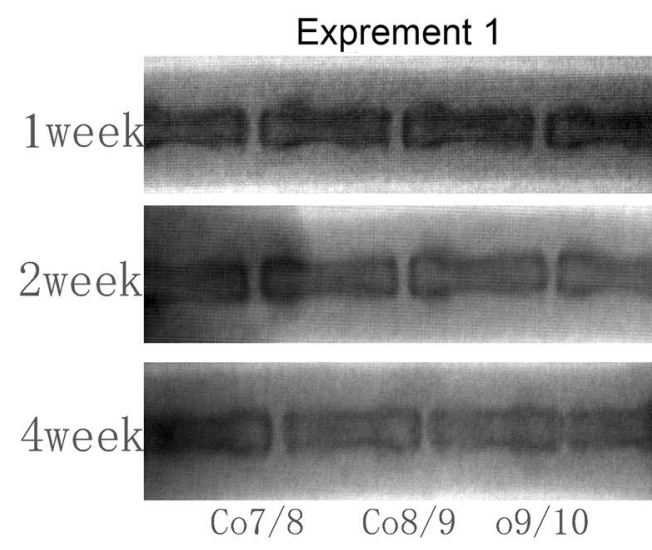

Exprement 1

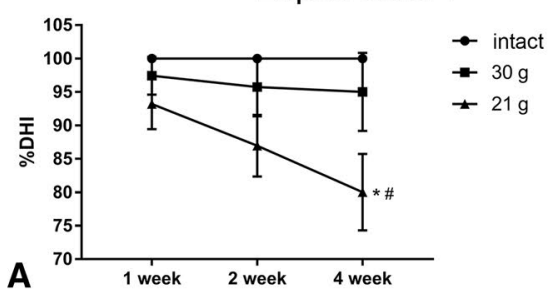

Exprement 2
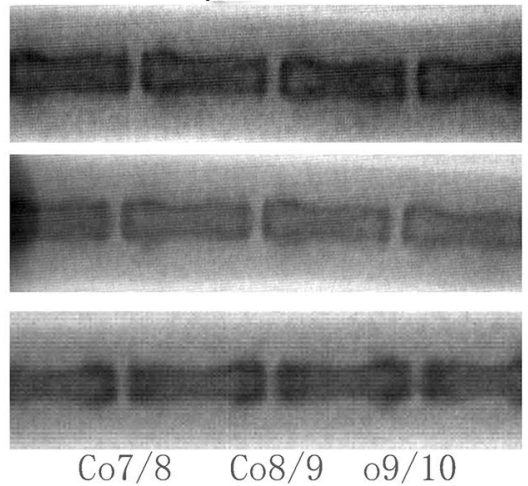

Exprement 2

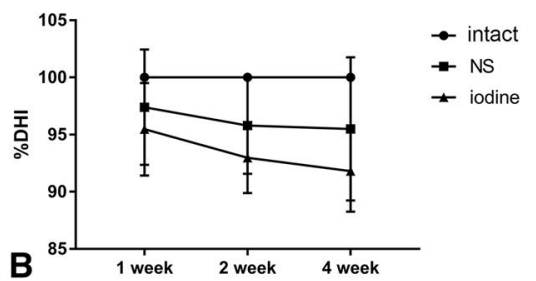

Exprement 3
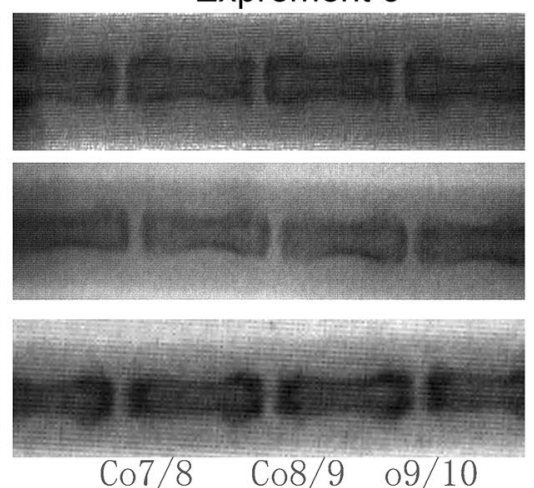

Fig. 2 Top: typical radiographs (top) and DHI ratio change (bottom) of the tail discs of the three experiments at 1,2 and 4 weeks after the procedures. The disc heights of discs that were operated on according to the experimental design shown in supplemental Fig. 1 were decreased at weeks 2 and 4 . The disc height index (DHI) of the $21-\mathrm{g}$ and $3.0 \mu \mathrm{l}$ discs differed significantly from that of the intact and $(30-\mathrm{g}, 2 \mu \mathrm{l})$ discs at 2 weeks and 4 . Data presented as mean \pm SD. $* P<0.05$ versus Co7/8 (intact); $\# P<0.05$ versus Co8/9 ( $n=10$ rats/ experiment) 

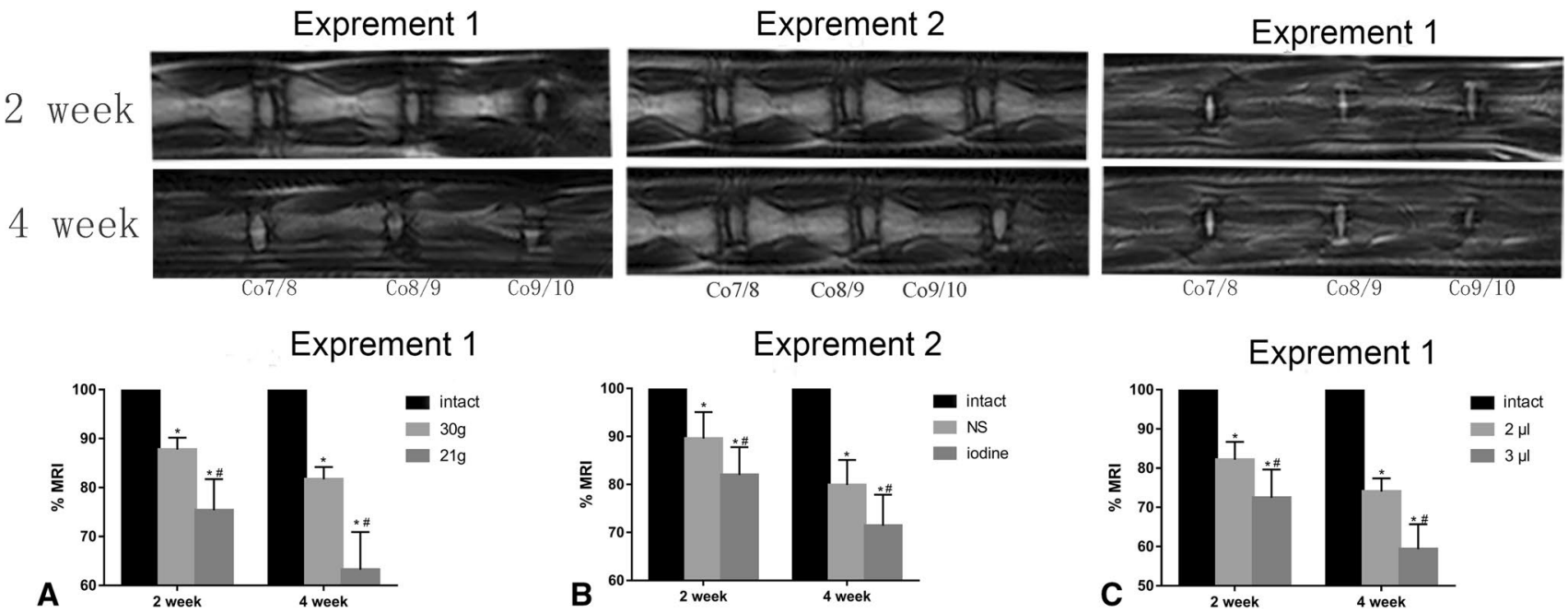

\section{Exprement 2}

\section{Exprement 1}
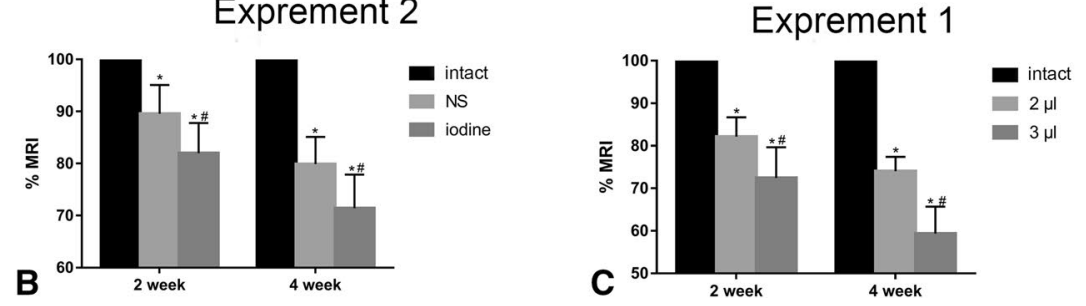

Fig. 3 Typical MRI and signal intensity of MRI ratio change in the tail discs of the three experiments at 1, 2 and 4 week after procedures. The disc MRI signal intensity of experiments that were operated according to the indicated experimental design was decreased at weeks 2 and 4 . The signal intensity of MRI ratio of the 21-g, iodine and $3.0 \mu \mathrm{l}$ discs differed significantly from that of the intact and $30-\mathrm{g}$, NS and $2 \mu \mathrm{l}$ discs, respectively, at weeks 2 and 4 . Data presented as mean \pm SD. $* P<0.05$ versus $\operatorname{Co} 7 / 8$; ${ }^{\#} P<0.05$ versus $\operatorname{Co} 8 / 9(n=5$ rats/experiment)
A

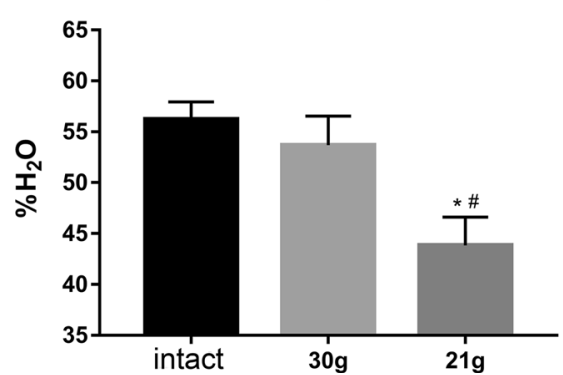

B

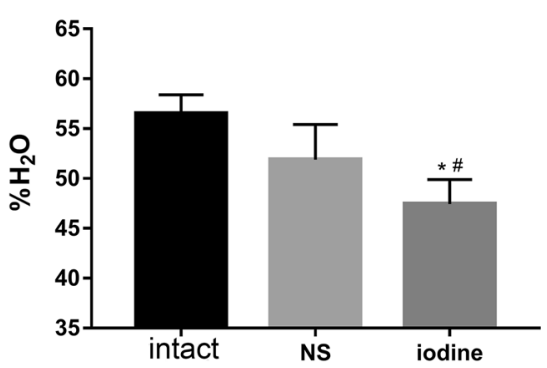

C

Exprement 3

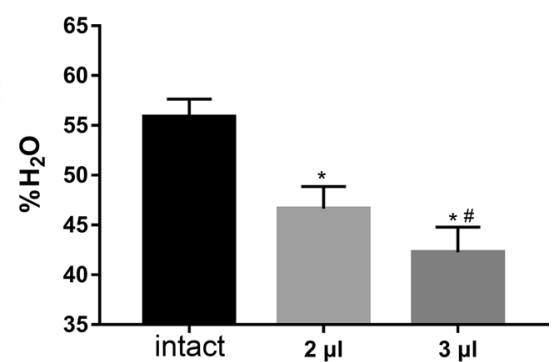

Fig. 4 The water content change in the tail discs of the three experiments at 4 weeks after indicated procedures. The water content of the $21-\mathrm{g}$, iodine and $3.0 \mu \mathrm{l}$ discs differed significantly from that of the intact and $30-\mathrm{g}$, NS and $2 \mu \mathrm{l}$ discs, respectively. Also, the water con- tent of $2.0 \mu \mathrm{l}$ discs differed significantly from that of the intact discs. Data presented as mean $\pm \mathrm{SD}$. ${ }^{*} P<0.05$ versus $\mathrm{Co} 7 / 8$; ${ }^{\#} P<0.05$ versus $\operatorname{Co} 8 / 9$ ( $n=5$ rats/experiment)

was much higher than that in the Co7/8 (intact) and Co8/9 $(2 \mu \mathrm{l})$ discs (Fig. 5, experiment $3, P<0.05$ ). These results demonstrated that if the volume of contrast agent exceeded a threshold, it rapidly exhibited degenerative changes. The degenerative changes were dose-dependent and increased as the dose increased.

\section{Discussion}

In this study, the effect of puncture needle size, type of contrast agent, capacity of contrast agent on disc degeneration in discography was studied in a rat model, which provided a theoretical and experimental basis for assessing the value 

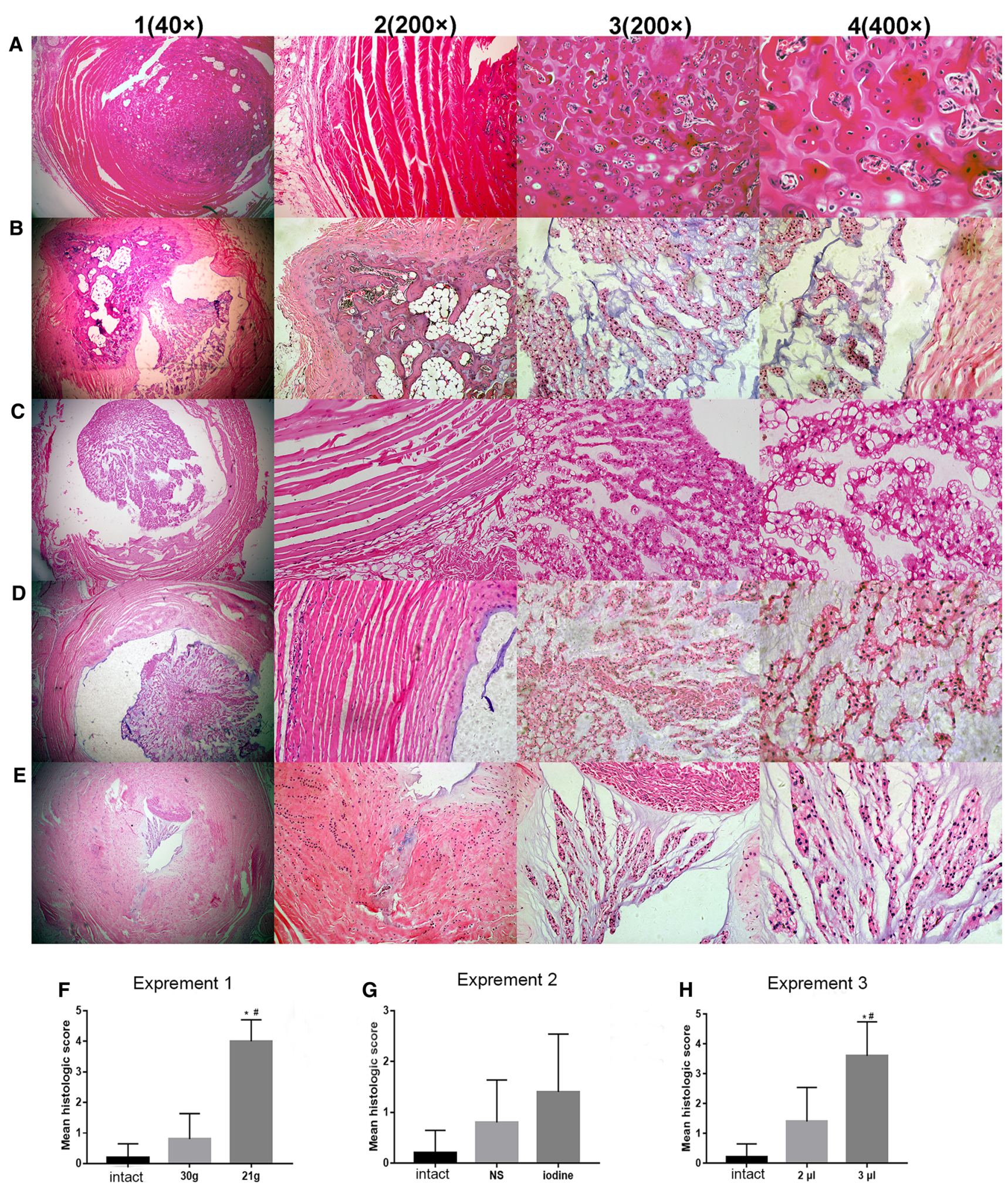

Fig. 5 Typical HE staining and histologic scores of the tail discs of the three experiments at 4 weeks after the procedures. a Internal intact disc, $\mathbf{b}$ internal disc injected with $2 \mu \mathrm{l}$ NS by 21 -g needle, $\mathbf{c}$ internal disc injected with $2 \mu \mathrm{l}$ NS by 30 -g needle, $\mathbf{d}$ internal disc injected with $2 \mu \mathrm{l}$ iodine by $30-\mathrm{g}$ needle, $\mathbf{e}$ internal disc injected with
$3 \mu \mathrm{l}$ iodine by $30-\mathrm{g}$ needle, $\mathbf{f}-\mathbf{h}$ histologic score changes. The histologic scores of the $21 \mathrm{-g}$ and $3.0 \mu \mathrm{l}$ discs differed significantly from that of the intact and 30-g and $2 \mu \mathrm{l}$ discs, respectively. Data presented as mean $\pm \mathrm{SD}$. $* P<0.05$ versus $\operatorname{Co} 7 / 8,{ }^{\#} P<0.05$ versus $\operatorname{Co} 8 / 9(n=5$ rats/experiment) 
and risk of clinical discography. In the first experiment, we found a significant difference in the degree of disc degeneration in the 21-g discs from that of the intact and 30-g discs at weeks 2 and 4 . This change was similar to those described in previous disc degeneration studies [7], but this study focused on the simulation of discography, so the same measurement of physiology was performed in different diameter needle groups. The injection of saline, therefore, has different factors affecting the intervertebral disc, increasing the influencing factors of intradiscal injection. In addition, the differences in laboratory animals, modelling methods, detection indexes and follow-up time were all different. In the second experiment, we found a significant difference in the degree of disc degeneration in the $2 \mu \mathrm{l}$ iodine discs compared to the intact and $2 \mu 1 \mathrm{NS}$ discs at weeks 2 and 4 . In the third experiment, we found a significant difference in the degree of disc degeneration in the $3 \mu \mathrm{l}$ iodine discs from that of the intact and $2 \mu \mathrm{l}$ iodine discs at weeks 2 and 4 . This conclusion is consistent with previous studies [23]. The difference between studies 3 and the previous literature is that this study focused on simulating the discography process, so iohexol injections were performed in different dose groups instead of the injection of normal saline.

Normally, we should first calculate the volume of the human intervertebral disc and then follow the volume for further injection. However, in clinical practice, we continue to inject until the patient has pain or the disc has been unable to continue the injection. The volume of the injection is generally from 0.5 to $1 \mathrm{ml}$. The mean disc height and nucleus area of an adult rat caudal disc are $0.94 \mathrm{~mm}$ and $3.30 \mathrm{~mm}^{2}$, respectively [24]. Therefore, the total nucleus volume of an adult rat caudal disc is $3.10 \mathrm{~mm}^{3}$. Studies have shown that in SD rats, each IVD injection of less than $2 \mu \mathrm{l}$ did not cause significant IVD degeneration [23]. Consequently, the adult rats in our study were injected with volumes of normal saline (NS) of 2 and $3 \mu$ l. Since the rat could not describe its pain, we used the calculated volume for the injection.

Iodine (trade name Omnipaque) is the second generation of non-ionic contrast commonly used in clinical discography. Studies have shown that contrast agents have a certain incidence of adverse reactions, including seepage, allergic reactions, alcohol nephropathy and neurotoxicity [25]. Yano reported that Cyclic AMP reverses radiocontrast media-induced apoptosis in LLC-PK1 cells by activating A kinase/-PI3 kinase [26]. In vitro cell studies have shown the toxic effects of iodide on renal proximal tumour cells and endothelial cells [27]. The cytotoxicity of different types of non-ionic contrast agents is different, which may be related to osmolality and viscosity of different contrast agents [28]. When more iodine was injected, there was more severe IVD degeneration, which was the same as the previously reported results of injection volume on disc degeneration in a rat tail model [23]. The IVD is the largest avascular tissue of the human body, and there is a special hypertonic environment from 430 to $496 \mathrm{mOsm}$ in the IVD, meaning that the injected iodine can remain in the IVD for a longer period of time. The degree of IVD degeneration is related to the hydrostatic pressure of the IVD. The iodine osmotic pressure is $780 \mathrm{mOsm} / \mathrm{kg}$ and much higher than the osmotic pressure of the disc, which leads to liquid extravasation in the nucleus pulposus, annulus fibrosus cells and cells damage $[6,28]$. In vivo and in vitro studies have clearly demonstrated that hydrostatic pressure affects the turnover of the matrix and the activity and gene expression of the cell metabolizing protein $[23,29,30]$. Our study found that when a greater volume of iodine was injected into a closed IVD, there was a more severe degeneration. One possible reason is that the greater the volume of iodine injection, the higher the hydrostatic pressure, and the more the necrosis of intervertebral disc cells, which ultimately leads to more serious IVD degeneration [31, 32].

This study suggests certain guidelines for the clinical application of discography. First, discography should be done with a smaller-diameter needle. Our experimental results showed that the 30 -g needle did not cause significant degeneration of the disc. Although Elliott et al. [7] reviewed that the effect of puncture on the IVD was variable and insignificant when the diameter of the needle was less than $40 \%$ of the IVD height, this analysis was limited by ex vivo tissue and only biomechanical testing. The idea that larger diameter of the puncture needle can significantly cause disc degeneration was controversial, because it is unknown what size needle begins to cause the degeneration $[3,15]$. The smaller the diameter of the puncture needle, the more difficult it was to puncture the disc, and needle breakage and an assortment of other risks increased. Therefore, we should choose the smaller-diameter puncture needle as far as possible without affecting the difficulty of discography and increasing the associated risks. Second, discography should try to use the minimum dose of contrast agent, which may cause pain, and use NS instead if necessary.

There were also some shortcomings in this study. First, this study investigated the effect of discography on the IVD only from the two aspects of the needle size and contrast dose. There are other factors that can cause the degeneration of discs, such as disc size (fibre ring thickness), operator proficiency, the nutritional status of experimental animals and other unknown factors. Secondly, the target of this experiment was a normal IVD, but the effect of discography on degenerated IVD was not studied. Using normal discs could avoid systematic errors due to variations in the baseline induced IVD degeneration. Thirdly, the study did not randomize the treatment levels. If each procedure of one group was performed at the same level and compared to another group with individuals from 
another level, the results and conclusions obtained will be more persuasive. Fourth, rats were used as the subjects in this experiment. However, because of the differences between the IVD nucleus pulposus cell morphology, physiological function and the osmotic pressure of the disc between human beings and rats, the iodine osmotic pressure-induced disc degeneration may be different. Further research on human IVD is needed.

\section{Conclusion}

The results of our study indicated that risks and benefits of the discography itself should be taken into consideration. Discography should try to choose a small needle size, minimize the use of contrast agents and try to use nonionic contrast agents with osmotic pressure similar to the intervertebral disc to reduce the impact on the intervertebral disc. The discography variables on rats IVD degeneration and validation in human IVD require further study.

Acknowledgements This project was supported by National Natural Science Foundation of China (Grant Nos. 81472071, 81301537), Military Medical Science and Technology Youth Cultivation PLAN of PLA (Grant No. 15QNP022) and Medical Science and Technology Innovation Project of PLA Nanjing military region (Grant No. 15MS107).

\section{Compliance with ethical standards}

Conflict of interest The authors declare that there is no conflict of interests regarding this paper.

Ethical standards All animal protocols were approved in accordance with the Association for Research in Vision and Ophthalmology guidelines and the Animal Care and Use Committee of Second Military Medical University.

Open Access This article is distributed under the terms of the Creative Commons Attribution 4.0 International License (http://creativeco mmons.org/licenses/by/4.0/), which permits unrestricted use, distribution, and reproduction in any medium, provided you give appropriate credit to the original author(s) and the source, provide a link to the Creative Commons license, and indicate if changes were made.

\section{References}

1. Collis JS Jr, Gardner WJ (1962) Lumbar discography. An analysis of one thousand cases. J Neurosurg 19:452-461

2. Walsh TR, Weinstein JN, Spratt KF, Lehmann TR, Aprill C, Sayre H (1990) Lumbar discography in normal subjects. A controlled, prospective study. J Bone Jt Surg Am 72:1081-1088

3. Carragee EJ, Don AS, Hurwitz EL, Cuellar JM, Carrino JA, Herzog R (2009) ISSLS prize winner: does discography cause accelerated progression of degeneration changes in the lumbar disc: a ten-year matched cohort study. Spine 34:2338-2345
4. Cuellar JM, Stauff MP, Herzog RJ, Carrino JA, Baker GA, Carragee EJ (2016) Does provocative discography cause clinically important injury to the lumbar intervertebral disc? A 10-year matched cohort study. Spine J 16:273-280

5. Strube P, Pfitzner BM, Streitparth F, Hartwig T, Putzier M (2017) In vivo effects of bupivacaine and gadobutrol on the intervertebral disc following discoblock and discography: a histological analysis. Eur Radiol 27:149-156

6. Chee AV, Ren J, Lenart BA, Chen EY, Zhang Y, An HS (2014) Cytotoxicity of local anesthetics and nonionic contrast agents on bovine intervertebral disc cells cultured in a three-dimensional culture system. Spine J 14:491-498

7. Elliott DM, Yerramalli CS, Beckstein JC, Boxberger JI, Johannessen W, Vresilovic EJ (2008) The effect of relative needle diameter in puncture and sham injection animal models of degeneration. Spine 33:588-596

8. Korecki CL, Costi JJ, Iatridis JC (2008) Needle puncture injury affects intervertebral disc mechanics and biology in an organ culture model. Spine 33:235-241

9. Michalek AJ, Iatridis JC (2012) Height and torsional stiffness are most sensitive to annular injury in large animal intervertebral discs. Spine J 12:425-432

10. Michalek AJ, Buckley MR, Bonassar LJ, Cohen I, Iatridis JC (2010) The effects of needle puncture injury on microscale shear strain in the intervertebral disc annulus fibrosus. Spine J 10:1098-1105

11. Iatridis JC, Nicoll SB, Michalek AJ, Walter BA, Gupta MS (2013) Role of biomechanics in intervertebral disc degeneration and regenerative therapies: What needs repairing in the disc and what are promising biomaterials for its repair? Spine J $13: 243-262$

12. Han B, Zhu K, Li FC, Xiao YX, Feng J, Shi ZL, Lin M, Wang J, Chen QX (2008) A simple disc degeneration model induced by percutaneous needle puncture in the rat tail. Spine 33:1925-1934

13. Masuda K, Aota Y, Muehleman C, Imai Y, Okuma M, Thonar EJ, Andersson GB, An HS (2005) A novel rabbit model of mild, reproducible disc degeneration by an anulus needle puncture: correlation between the degree of disc injury and radiological and histological appearances of disc degeneration. Spine 30:5-14

14. Sobajima S, Kompel JF, Kim JS, Wallach CJ, Robertson DD, Vogt MT, Kang JD, Gilbertson LG (2005) A slowly progressive and reproducible animal model of intervertebral disc degeneration characterized by MRI, X-ray, and histology. Spine 30:15-24

15. Issy AC, Castania V, Silveira JW, Nogueira-Barbosa MH, Salmon CE, Del-Bel E, Defino HL (2015) Does a small size needle puncture cause intervertebral disc changes? Acta Cir Bras 30:574-579

16. Fazzalari NL, Costi JJ, Hearn TC, Fraser RD, Vernon-Roberts B, Hutchinson J, Manthey BA, Parkinson IH, Sinclair C (2001) Mechanical and pathologic consequences of induced concentric anular tears in an ovine model. Spine 26:2575-2581

17. Kirberger RM, Cassel N, Carstens A, Goddard A (2012) The effects of repeated intravenous iohexol administration on renal function in healthy beagles: a preliminary report. Acta Vet Scand 54:47

18. Gruber HE, Rhyne AL 3rd, Hansen KJ, Phillips RC, Hoelscher GL, Ingram JA, Norton HJ, Hanley EN Jr (2012) Deleterious effects of discography radiocontrast solution on human annulus cell in vitro: changes in cell viability, proliferation, and apoptosis in exposed cells. Spine J 12:329-335

19. Michalek AJ, Iatridis JC (2011) Penetrating annulus fibrosus injuries affect dynamic compressive behaviors of the intervertebral disc via altered fluid flow: an analytical interpretation. J Biomech Eng 133:084502

20. Silveira JW, Issy AC, Castania VA, Salmon CE, Nogueira-Barbosa MH, Guimaraes FS, Defino HL, Del Bel E (2014) Protective 
effects of cannabidiol on lesion-induced intervertebral disc degeneration. PLoS ONE 9:e113161

21. Lin Y, Li F, Chen W, Zeng H, Chen A, Xiong W (2015) Singlelevel lumbar pyogenic spondylodiscitis treated with mini-open anterior debridement and fusion in combination with posterior percutaneous fixation via a modified anterior lumbar interbody fusion approach. J Neurosurg Spine 23:747-753

22. Pfirrmann CW, Metzdorf A, Zanetti M, Hodler J, Boos N (2001) Magnetic resonance classification of lumbar intervertebral disc degeneration. Spine 26:1873-1878

23. Mao HJ, Chen QX, Han B, Li FC, Feng J, Shi ZL, Lin M, Wang $J$ (2011) The effect of injection volume on disc degeneration in a rat tail model. Spine 36:E1062-E1069

24. O'Connell GD, Vresilovic EJ, Elliott DM (2007) Comparison of animals used in disc research to human lumbar disc geometry. Spine 32:328-333

25. Yeganehkhah MR, Iranirad L, Dorri F, Pazoki S, Akbari H, Miryounesi M, Vahedian M, Nazeri A, Hosseinzadeh F, Vafaeimanesh J (2014) Comparison between three supportive treatments for prevention of contrast-induced nephropathy in high-risk patients undergoing coronary angiography. Saudi J Kidney Dis Transpl 25:1217-1223

26. Yano T, Itoh Y, Sendo T, Kubota T, Oishi R (2003) Cyclic AMP reverses radiocontrast media-induced apoptosis in LLC-PK1 cells by activating A kinase/PI3 kinase. Kidney Int 64:2052-2063

27. Heinrich M, Scheer M, Heckmann M, Bautz W, Uder M (2007) Reversibility and time-dependency of contrast medium induced inhibition of 3-(4,5-dimethylthiazol-2-yl)-2,5-diphenyl-tetrazolium bromide (MTT) conversion in renal proximal tubular cells in vitro: comparison of a monomeric and a dimeric nonionic iodinated contrast medium. Invest Radiol 42:732-738

28. Duarte CG, Zhang J, Ellis S (2001) Review of studies establishing the aging male spontaneously hypertensive rat as a detector and quantifier of the kidney toxicity of radiocontrast media and other chemicals. Invest Radiol 36:56-63

29. Chen J, Yan W, Setton LA (2004) Static compression induces zonal-specific changes in gene expression for extracellular matrix and cytoskeletal proteins in intervertebral disc cells in vitro. Matrix Biol J Int Soc Matrix Biol 22:573-583

30. Hutton WC, Elmer WA, Bryce LM, Kozlowska EE, Boden SD, Kozlowski M (2001) Do the intervertebral disc cells respond to different levels of hydrostatic pressure? Clin Biomech (Bristol, Avon) 16:728-734

31. Panjabi M, Brown M, Lindahl S, Irstam L, Hermens M (1988) Intrinsic disc pressure as a measure of integrity of the lumbar spine. Spine 13:913-917

32. Pezowicz CA, Schechtman H, Robertson PA, Broom ND (2006) Mechanisms of anular failure resulting from excessive intradiscal pressure: a microstructural-micromechanical investigation. Spine 31:2891-2903

Publisher's Note Springer Nature remains neutral with regard to jurisdictional claims in published maps and institutional affiliations.

\section{Affiliations}

\section{Xiaodong Huang ${ }^{1} \cdot$ Weiheng Wang $^{1} \cdot$ Qingxi Meng $^{1} \cdot$ Lei Yu ${ }^{1} \cdot$ Chunquan Fan ${ }^{2} \cdot$ Jiangming Y $^{1}{ }^{1} \cdot$ Mintao Xue $^{1}$. Xiaojian $\mathrm{Ye}^{1}$ (1)}

Mintao Xue

xmt1984@smmu.edu.cn

Xiaojian Ye

xjyespine@smmu.edu.cn

1 Department of Orthopaedics, Changzheng Hospital, Second Military Medical University, 415 Fengyang Road, Shanghai 200003, China
2 Department of Orthopaedic Surgery, The 175th Hospital of PLA, Orthopaedics Center of PLA, Affiliated Southeast Hospital of Xiamen University, Zhangzhou 363000, Fujian Province, China 